\title{
ELECTRONIC MARKETS ONTOLOGY: IDEAL ARCHITECTURE FOR A GLOBAL CAPITAL MARKET
}

\author{
Davide Khalil \\ Edinburgh Business School \\ Heriot-Watt University \\ Edinburgh, Scotland EH14 4AS \\ Email: nine@cyberservices.com
}

\begin{abstract}
When approaching electronic capital market design and microstructure with the focus of analysing and improving existing markets with end-state analysis, it is necessary to name an ideal objective. This serves the purposes of technology evaluation and the development of a standard framework for structural measurement in modeling and language paradigm design. An ideal capital market architecture is presented in this paper that is feasible with current technology based on the end-to-end functionality of existing capital markets including internal requirements of participants. Various architectural and ethical issues are introduced and discussed sketching a framework for further work in quantifying electronic markets.
\end{abstract}

\section{INTRODUCTION}

Electronic capital markets have been developing since the systematic, commercial use of high-speed computers began in the 1970's. With the end of the Cold War and the spread of global capitalism, nations throughout the world are attempting to install and develop capital markets. In the engineering of a capital market software system, the marketplace microstructure codified into the software is reflected in the real world in terms of the efficiency of the market and in implicit advantages for certain market participants (Cohen, 1986). To evaluate these influences an end-state model is proposed for objectives and ideals with which technologies can be described and measured for their suitability of purpose and quality (Prindl, 1994). This process involves sifting through the entire set of requirements and perceived values currently provided by all stakeholders in a capital market (Fabozzi, 1996). A simplified analysis is presented as a root model.

An electronic capital market is a virtual version of a physical market. There are goods for sale, buyers, sellers, the auction process and agents of the market. The primary object moving in and out of the market is cash. Unlike other marketplaces, the goods for sale are delivered virtually and converted to cash only if the entity wishes to take them from the market with the exception of certain contracts where delivery of physicals is part of the contract. Every dealing party is concerned with their inventory of goods, bringing goods to market, the auction market for the goods, qualities of the goods themselves and their membership status and accounts.

Because no entity in the marketplace keeps consolidated information as a service, each entity keeps its own record of the portion of data that is relevant to its own needs and thus spends enormous sums on information technology to maintain what is already recorded elsewhere in the marketplace. Over years, each entity's interests with this data has lead to specialised representations to each user community on a need-to-automate basis (Reddy, 1995)(Pardo, 1992)(O'Leary, 1997). This has lead to databases throughout the industry keeping a patchwork of data, none of which is an absolute for a marketplace. These factors have spearheaded a specific demand from computer science in the development of artificial intelligence software ontology's for the representation of a whole trading market. In effect, the knowledge demands of the various dealing parties are optimally represented in an ideal virtual market ignoring the locality of the underlying corporate entities.

The ideal market model presented is designed to provide the optimum marginal benefit for all market participants. This ideal is considered to be a multi-image tightly distributed simultaneous ${ }^{12}$ global capital

12 A simultaneous market actually exchanges value at the moment of trading. There is no settlement delay as all payments and transfers happen at the point of exchange. In order to have a simultaneous market; the depository and other aspects of the "processing" of today's trade are the execution process itself. A simultaneous market is the ultimate evolution of the current settlement system. It also greatly simplifies issues in modern computerized finance to dispense with the delay between front office actions and actual accounts. For these reasons, an ideal market is a simultaneous market. 
market on a high bandwidth secure network with images in every sovereign constituency. The model is first discussed in terms of its selection and is then broken down into a top-level software paradigm that could be constructed using a variety of underlying physical hardware and software sub-systems such as supercomputers and databases (example in Appendix A). This is composed of a market engine, required media services and network services to support such an ideal global market. The market knowledge required to support these services is presented along with workflow ontology to bring together the various electronic market functions into a discrete market "instruction". The further ontological development of each portion of the market is outlined including its requirements as a basis for ongoing work.

This paper can be expected to be of interest to the IS community helping to formalise the joint discipline of electronic markets as a field involving finance, accountancy, economics, law, ethics, telecommunications, computer science and industry current practices. This broad knowledge scope combined with the vertical structure of university departments and disciplines has restricted research in this area. Further research will yield models of optimal economic markets capable of testing various market structures for advantage as well as models for re-engineering the business in marketplace media, capital markets themselves and in financial organisations. In the macroeconomic sense, it is possible to introduce a capital markets efficiency and effectiveness multiplier that can geometrically stimulate economic output though further innovation along the lines of the technologies described in this paper.

Within the software domain, design parameters can vary quite widely to accommodate an optimal representation. The following conceptual model (figure 1) is presented in simplicity to keep the paper short and to facilitate comprehension of a broad domain of knowledge. Were a further notch of real-market complexity introduced, this paper would develop into an extremely complex software design.

\section{POSTULATES \& ASSERTIONS}

There can be significant debate among economists on what qualities represent an ideal market. The following 18 postulates are underlying assumptions underlying subsequent arguments.

1. An ideal market maximises the marginal benefit of each entity connected to the marketplace and it is in their best interests to participate in the marketplace even if a dealing party has an absolute advantage in the production of goods (Smith, 1776).

2. Ideal market governance is representative democracy of its member constituents and operated under no single sovereign, thus no agency motivation from the directors. ${ }^{13}$

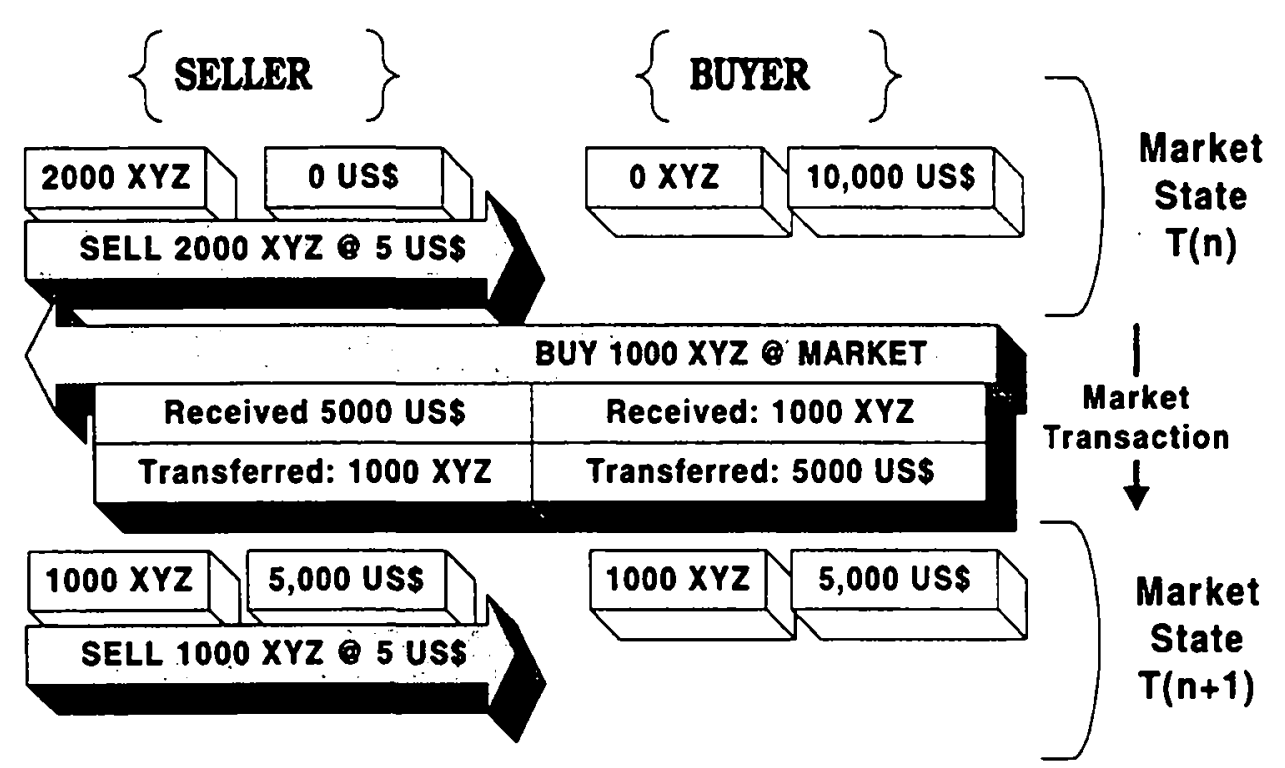

Figure 1. Note how a simultaneous market is always in a state of equilibrium. When a matching event occurs, the market changes state and again resumes a state of equilibrium.

13 The definition of directorship is limited to reasonable representation or accepted asymmetry by capitalization. Below a certain capitalization threshold, there is no marginal benefit for participation in a capital market and thus no representation in the directorship. 
3. An ideal capital market has minimal barriers to entry. This includes the cost of the connection to the marketplace and the knowledge and comprehensibility of the market structure which is a more likely realworld barrier-to-entry.

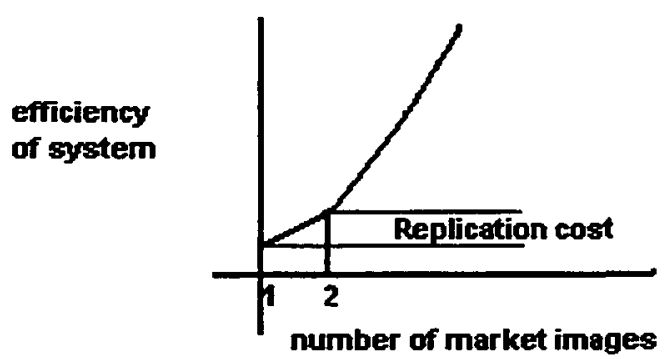

4. (ASSERTION) One single large distributed system is the ideal market. Replicated systems with heterogeneous software environments introduce inefficiency to an ideal market.

The replication cost is perpetuated due to:

a) The replication delay in maintaining multiple equivalent data images and the additional network traffic of that replication.

b) Human error factor forcing systems to reconcile through manual processes.

c) Market technology evolutions involve risk and the nature of finance is risk-adverse.

d) Conflict-of-interest's in the IS industry as the business model for profitability in software is based on quantity of installations or licenses sold, not efficiency of end-application function.

e) Successful standards come from small groups or individuals largely due to the intensity of knowledge that must be brought together to innovate new standards (Kahin, 1995). (e.g. UNIX, C, $\mathrm{C}++$ )

5. An ideal capital market is completely secure through the intelligent use of encryption, secure physical access, and policing (Marinelli, 1991).

6. Clean-slate consideration - Any knowledge systems innovated prior to the development of global networks and modern supercomputing are accepted in terms of their essential discipline and not in terms of the methodologies themselves. This is especially true of procedural computer science, but also in accounting (Kam, 1986), law (Susskind, 1996), economics, current business process (Reddy 1995) and finance (Arbnor, 1997).

7. There is one time zone for a simultaneous market. A global market is operating across time zones. Current telephone networks have the same consideration and use "Zulu time" as a geocentric time (Bellcore 1984).

8. An ideal market does not have an asymmetry in favour of any one entity. Many existing capital markets have asymmetries in favour of the well-capitalised dealers and customers, sovereign nations or exchanges themselves. Though it is the standard practice, asymmetries only weaken a market and for the body of the paper make the assumption that neutral asymmetries improve the climate for frictionless exchange (Prindl, 1994)(Cohen, 1986).

9. Markets are always open. The open/close times of regional markets are related to the human resources involved in operating the market. A global frictionless Computerized market has no such concerns. It is also to the benefit of all connected parties if news expectations are simultaneously realised in the market rather than forcing up volatility expectations for listed instruments during that time that markets are closed.

10. An ideal capital market is instantaneously replicated to multiple secure physical sites to avert concerns over risks of catastrophe and sovereign control. For a quantifiable replication cost in terms of system complexity, multiple images of a complex system can be maintained (Tandem, 1989).

11. A capital market's software does not make mistakes. Though not the case over the past 25 years, it is possible to create highly complex systems that are error-free for their function as they are tested so well. As of today, these include space vehicle computers, the telephone network in western nations, bank ATM machines and military systems. 
12. An ideal market facilitates the development and fair pricing of financial products by making them simpler to bring to market and easier to disclose and measure.

13. An ideal market is simultaneous and has no settlement risk or contraparty credit risk as all transactions clear before acknowledgment of execution.

14. An ideal market has minimum execution risk. Every participant may enter the market with confidence that they will get the best price from the ideal market. This is a function of liquidity and the design of the market auction process (Optimark, 1998).

15. An ideal market is not taxed internally, deriving all internal operating revenues from connection charges. This "commission" issue is a major source of complexity in existing market systems (Reddy, 1995).

16. An ideal capital market supports the interests of both clever participants and innocent participants, loosely defined in the current marketplace as sell-side and buy-side (Prindl 1994)(Boyd, 1997).

17. An ideal capital market has a user-interface that has no learning curve and that allows each user to execute their business efficiently and effectively (Barber, 1997).

18. Human interests are best served by a government that abstains from interference in free enterprise, putting checks only on undue strife and competition (Smith, 1776).

\section{SIMULTANEOUS FRICTIONLESS MARKET}

Financial theory uses the concept of a "frictionless market", Walrasian auction, when considering ideal market valuation across changes in supply and demand. The following section describes a frictionless market in terms for a computer model of an "ideal" in modeling and language design. The market described here is technologically feasible today and is derived in function from today's capital markets. As the model argument is developed, it departs from the physical model of the market into an abstract world where a transaction can take place simultaneously. An electronic financial market is always in equilibrium. Value is never in transit; it is always accounted for. (Kam, 1986) Such a world is represented differently. The paper refers to this "different" representation as a market state of knowledge. A simultaneous market is the official accounting record of ownership from moment to moment as events change the market.

\section{Stakeholder scope}

The classic definition of a frictionless market includes its end users, the marketplace participants. An electronic market has a less visible set of stakeholders with widely varying requirements that must be considered for an ideal capital market architecture (Freixas, 1997). These stakeholders are:

1. Financial Users

2. System operators

3. Network administration.

4. Backup/recovery.

5. Legal

6. Accounting

7. Taxation agencies

8. Software development \& test (upgrade migration)

\section{Abstract meta-parameters}

Market design has a broad range of parameters that become implicit within the capital market architecture. The following parameters are thematic influences that must considered either deliberately or endemically in the architecture definition.

1. Aggregation - The unitary level at which every object interacting in the market is recognised.

a) Should the market keep record of individuals or just corporations.

b) Should it be easy or difficult to aggregate cash flows and instruments to create new products?

2. Complexity - What level of complexity should be presented within the market?

a) The user interface be so complex that only highly trained experts can use it. 
b) Should the market architecture be intuitively obvious to any educated person?

3. National Influence - The relationship between the sovereign and the market

a) The market is an extension of a sovereign legal and political system.

b) The market is country-independent and is not directly influenced by sovereign entities.

4. Liquidity - The market can be designed to increase or diminish participation.

a) Should every component of a market be fit for end-user facility?

b) Should the order-match process incorporate specialist-type liquidity insurance?

5. Capitalisation - What is the effect of capital leverage on the fairness of the market?

a) None. Each participant has the same transaction charges for participation removing all non-capital related barriers-to-entry for arbitrage.

b) Very high. Firms with more money should be able to purchase reasonable advantage in a marketplace with their leverage.

c) Cronyism. Capital leverage can buy leverage depending on its relationships.

6. Governance - What is the fairest way to control a market and its legitimacy?

a) Virtual Nation - The capital market is its own country with participants as citizens

b) Hegemony - One country runs the market for others

c) Plutocracy - One vote for one unit of capital leverage

d) Democracy - Each human being affected by a capital market has a vote in the market

e) Computocracy - The electronic capital market software is self-governing.

7. Privacy - What is the trade-off between the marketplace interest and participant liberty?

a) No market-internal information is ever disclosed (Mill, 1859).

b) Only reasonable disclosures to the public with total disclosures made to surveillance

c) All market information is public to all members

\section{Capital market knowledge domains}

Transactions in a market currently take days to propagate from exchange computers to the other systems in the broker-dealer chain that make up the current capital markets from initiation of deal to settlement. (Reddy, 1995) This data is marketplace domain knowledge and the delay is caused by antiquated computer and business infrastructure. (Jang, 1998) In a simultaneous architecture, representing all data in a single environment sustaining all requisite knowledge functions eliminates any propagation delays.

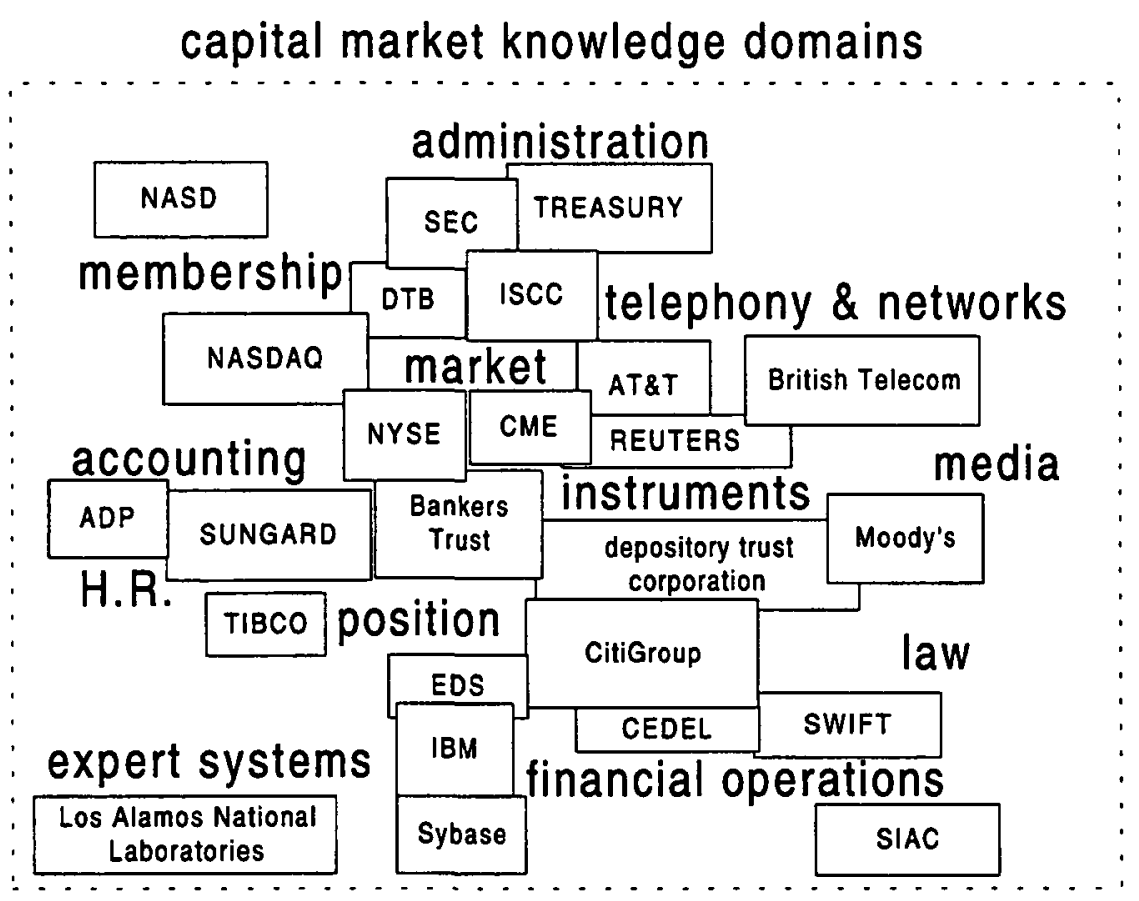

Figure 2. Companies with their market segments shown in reference to the knowledge areas in commercial capital markets. The dotted line encloses the scope of this frictionless market. The number of corporate barriers that are crossed to form a capital market is so large, that different parts have developed different ontologies for the same market data representation. In automation terms, they all process the same information, but break it up and view it differently. 


\section{Reference Frame}

Each entity involved in the market uses a common, underlying reference frame of knowledge (illustrated in Figure 3) to participate in that market. In this reference frame, there are certain archetypal bodies of knowledge that have standard interrelated properties. (Xticket, 1995) To computerise such a market, the systems representation must accurately model the market-state knowledge in each domain. Below is an illustration of how these domains are represented in the software architecture of a capital market.

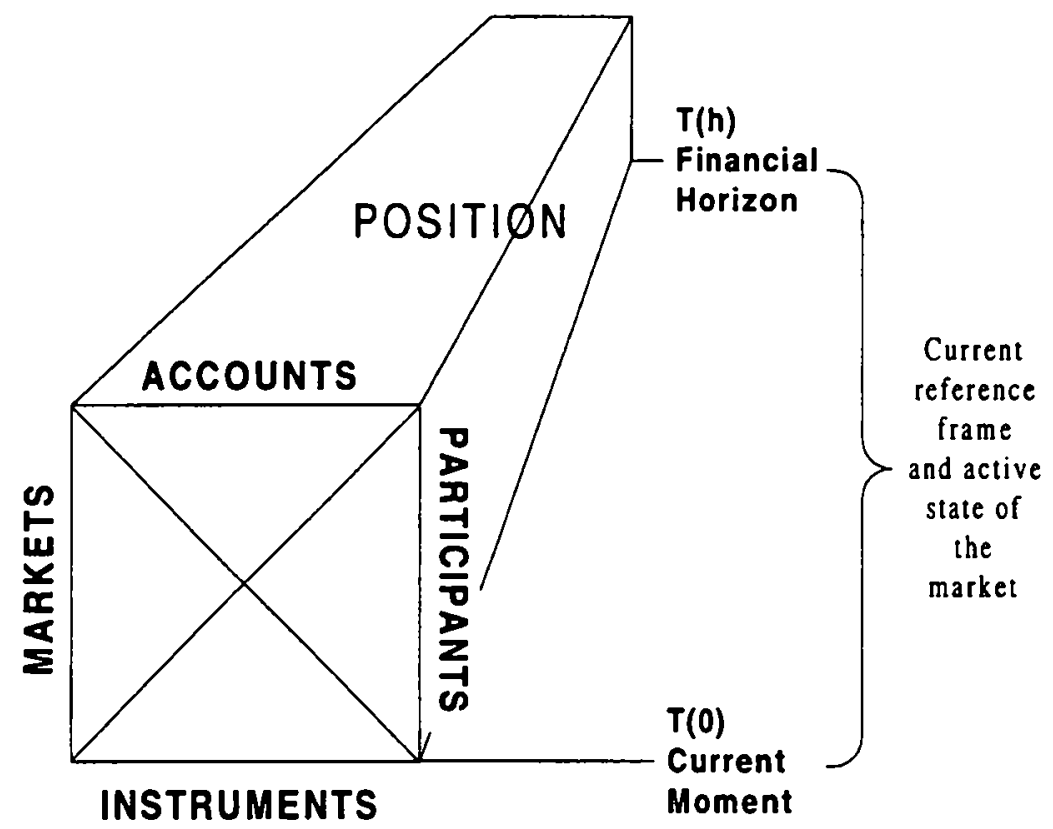

Figure 3. The current frame of the market is a joint representation of the state of accounts, members, instruments, markets and positions all interlocked in a state of exchange. The net-market position extends to a future time horizon beyond which no products are leveraged.

\section{Interrelationship}

The "market $x$ account $x$ instrument $x$ participant $=$ position" equation of the reference frame is the core abstract structure of a capital market. By keeping perfect reference-data integrity within such a logical marketplace, reconciliation and the repetitive back-office operations of the past can be eliminated. (OM Gruppen AB, 1996 , Nicolle, 1998) The ultimate critical result of isolating the market equation within the boundaries of pure-data market, however, is that the market can be safely driven by a discrete set of end-user instructions without risk of corruption or failure.

\section{Snapshot Market Images}

The market is referenced in either its current state, past states and by changes between states. Figure 3 illustrates only the current state. Any number of market images and derivative data can be maintained and generated from the Market transaction log for reporting and accounting purposes.

\section{Marketplace objects}

A marketplace is a body of expert system knowledge that participants must interact with to obtain their objectives. Based on this definition, the knowledge bodies that compose a marketplace are visible in Figure 4 . Each of these "objects" has an active state representing the current moment and the capability of showing historical states and the transaction logs. All messages that do not actually change the state of the market, are parts of what this paper calls marketplace media services. Events such as "checking an account balance" or 
"checking the P\&L in account A-I" do not change the market and thus are not market commands. The underlying objects in the diagram are each described in a subsequent section.

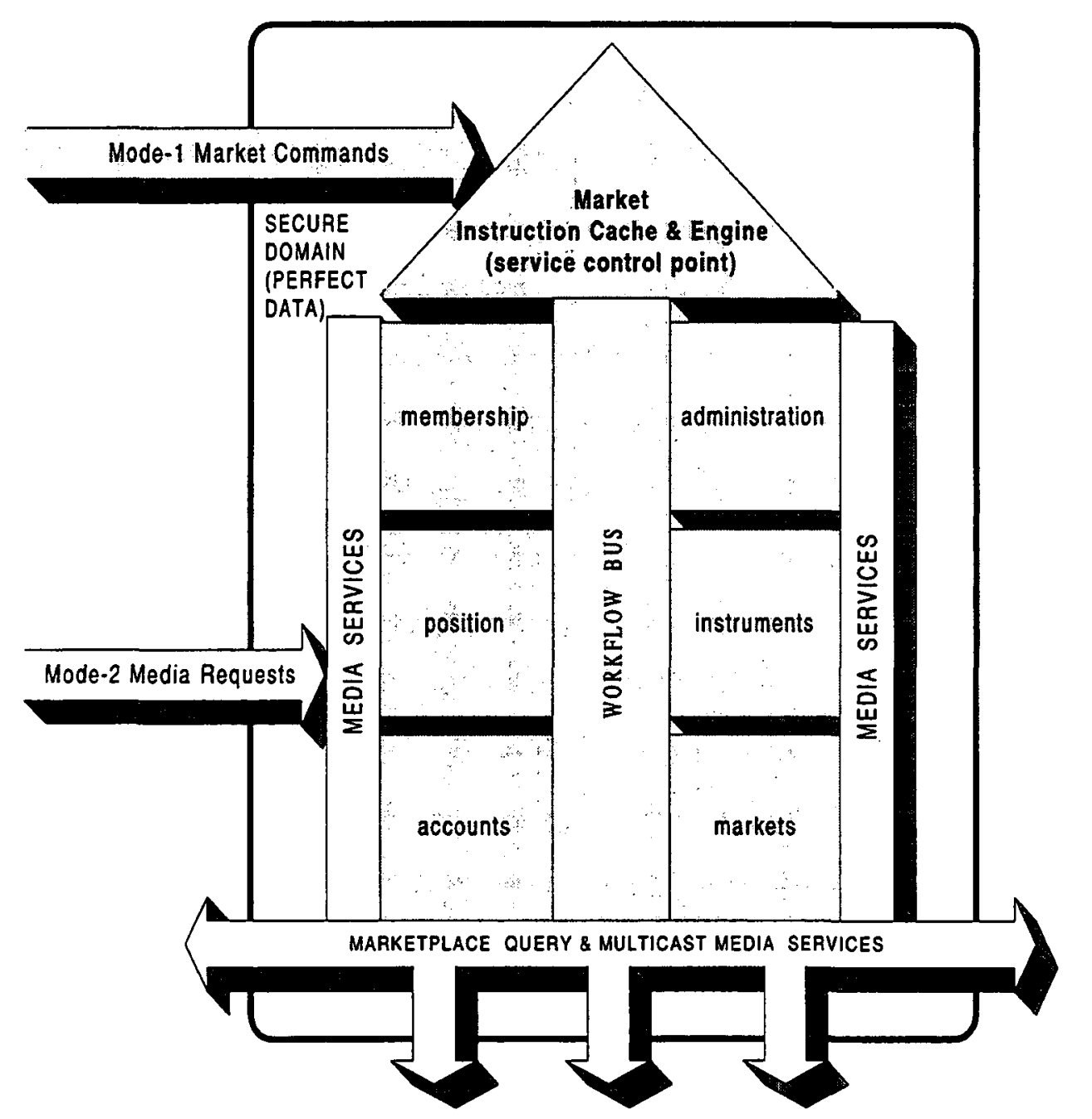

Figure 4. The box represents the main intelligence engine of a simultaneous market. Media services provide channels for global distribution and query regarding all aspects of the market. Market commands are actual instructions to buy, sell, open accounts or other actions that cause the market to change. The existing financial services industry operates similarly per the diagram except the workflow processes are implicit in the physical computer-to-computer connections between systems and in the manual processes of the marketplace.

\section{Membership}

Every connection to a market is defined as "membership". One broker dealer could have 15000 connections. The process of connecting to a capital market begins with membership. Although there are significant aspects of membership that involve non-market aspects of finance (e.g. human resources), they do not affect the electronic market internals. For an efficient electronic market, membership is considered similarly to telephony standard practices of the "service order". (Wolfe, 1988) (D'Sylva, 1991). The services that each member has access to, their billing status for marketplace services and all related support information are kept in the object. Easily a large and highly complex database, the object nevertheless contains only membership information for electronic processing purposes. There are types of market commands and hence "messages" that affect membership:

a) Query Member - Inquire membership information, permissions, limits, etc.

b) New Member - Add a new market connection

c) Change Member - change services, address, permissions, etc.

d) Delete Member - Disable member connection

e) Timer - Period based membership functions 
membership also includes the individuals, corporate entities and non-entities that access the market on behalf of an institution. This membership object also contains the security and permissioning for the market.

\section{Instruments}

The financial products traded in the marketplace are kept in a depository and virtually transferred by their entry in the instrument database object. This object represents all knowledge about the products including special issuance criteria and a market standard valuation algebra for derivative products. Instruments issue cash flows to their owners. Such instructions are driven by the instrument object automatically by a timer as in the case of a bond coupon payment. In the case of variable payment such as a dividend or a call on an issue of callable bonds determined by sources external to the marketplace, the instrument command is issued like an order as a marketplace instruction. Instruments each have an issuing party that is responsible for such instructions. Instrument messages:

a) Query Instrument - Inquire regarding the instrument

b) New Instrument - The instrument has passed physical criteria for depository and is being formally introduced

c) Change Instrument - call bonds, pay dividend (notifies marketplace media of relevant instrument change).

d) Delete Instrument - All positions must be flat and instrument is disabled

e) Timer - This marketplace command is issued by the instrument object timer itself for coupon payments and other internal "built-in" payments.

In order to trade an instrument unambiguously, a market transaction must have accurate instrument reference data. The instrument object must also be capable of representing newly engineered off balance sheet products without which, an ideal market could not exist. It is my position that ANY instrument including its valuation criteria can be represented using a quantum algebra involving expert system rules, and a "molecule" from a set of quantum finance "particles" including: time, probability, and value. In order to list the instrument, the financial dealer would have to use a published legal financial standard algebra and program the instrument.

\section{Accounts}

An account is a logical grouping known in the marketplace to hold a position. Each member will have many accounts and control the respective permissions for access, limits and transfer rules. For managed accounts, Allocations are a method within today's markets for electronically managing account inventories (FIX, 1998). The ownership of all the instruments on the market is distributed between the accounts, but not kept in the account object as that is a position, not an account. For these purposes, cash is considered a "position in a currency instrument" rather than a "balance in an account". Much of the media services reporting uses accounts as a driver to produce position and activity reports. Since all member inventory in the market is held in accounts, accounts are implicit in every market transfer transaction. Account messages:

a) New Account - Create new account for member

b) Change Account - Address, reporting-currency, permissions, allocation scheme, etc.

c) Delete Account - Verify that account has no position and retire account

\section{Position}

A position is financial leverage in the market. In practice, it is the marketplace inventory of financial instruments. (e.g. 2 Yen, 4 Dollars, 3 bonds, 1 digital option) The position is the current quantity of instruments held and the related transactions outstanding, including the purchase price(s), that created the position, but not the current market price. A buy order for IBM at 90 dollars per share with a time-in-force of 1 month from yesterday is also a position. Like an option, an order is a leveraged market commitment and is treated as such. All attempts to measure the value of marketplace holdings are part of assessing a position and thus incorporate all $\mathrm{P} \& \mathrm{~L}$ and time-based profitability \& risk media as requisite functionality of the market position-media services. Position messages:

a) Buy/SelVCancelreplace/Exercise/Cancel - Change position in the market

b) Allocate, BOT/SLD off-market

c) Deposit/Withdrawal - Import/Export cash from the market 
A number of buyers currently wants to buy. a number of sellers currently wants to sell of the same instrument. The market in an instrument is inseparable from the instrument itself as the bid-offer pool represents supply and demand for the instrument and related accounting values of corresponding positions. Order match including market transfer of ownership in goods takes place when the conditions of the seller's offer agree logically with the conditions a corresponding buyer's offer. (McKendrick, 1990) Conditions of entry, status and interconnection exist in this market space that the order match takes place in a [random - ordered] way. (Bernstein, 1995) If it is purely random, patterns of matched orders Vs the mean of the current pool of outstanding orders with the same order match criteria will show randomness. If it is ordered, then the market has asymmetries which are the focal point of marketplace design. A market is an expert system with the current frame as all buy and sell orders for a particular instrument. When a new fact is asserted (a new order), the system evaluates the current orders to see if there is a match. If so, then order match has taken place and the object issues a market command to transfer. If not, then the order is added to the current frame. Market messages:

a) Suspend/Resume - Market surveillance action stops all order match in an instrument. (Debatably, this is a sovereign asymmetry and should not be considered as root functionality.)

b) Timer-Order XX has reached its time-in-force without executing, delete.

A flexible systems architecture would support multiple interchangeable auction processes as part of the market object. The result would be that the market object is sophisticated and can support real requirements rather than enforcing any one set of auction market rules.

\section{Period of offer}

Current markets use a Time-in-force (TIF) in their order-match systems (NYSE, 1998). This is the life of an order in the market. This property gives a limit order the quality of an option contract. From a software point of view, there is no difference between a financial instrument and an order, leading one to consolidate non-OMS ${ }^{14}$ details regarding orders into the instrument metabase rather than the market metabase.

\section{All-or-none (AON)}

Some exchanges offer an order attribute that means an order is never broken up. (Rosenburg, 1987) If you are a small dealer, you may not be able to match the order quantity of a large order. Thus, the best market may not be available depending on capitalisation. Without the rule, an order for sale of 1000 at 40 per share would sell 500 to a buyer of 500 at 40 per share. Such a rule is a market asymmetry albeit a small one on behalf of larger capital institutions. This type of rule allows markets to cross (where the best bid is higher than the best offer). In a newer order match system such as Optimark (Optimark,1998), crude parameters like AON are replaced by urgency and price-sensitivity indicators clearly advancing along the line of allowing an end-user to express their strategy effectively and efficiently to the market. Nevertheless, the result is a market message described by quantitative parameters to be processed by a market order-match.

\section{Market Entry Triggers}

Current order-match environments offer the concept of an order that only becomes active contingent on an event within the market such as the last-sale price. The design of these trigger rules in an order-match environment, combined with the block sizing and order-breakup rules of the market are pivotal to the efficiency of the ordermatch system itself. Since orders "flow" at a certain rate of "traded-volume/second" (CME, 1997), a measurable standard flow moves in a market. To place orders into the flow without disturbing its price or getting a fair price in the flow are objectives to the end user.

\section{Administration}

Often implicit in the human business processes related to a market, the electronic market, network and software have innumerable issues that impact the state of the market. These include operations, billing, utilisation auditing, startup, shutdown, performance tuning, surveillance, servicing member requests and other actions regarding the marketplace that ultimately affect the market state (KSE, 1996). Under some circumstances, these activities can change the state of the market.

a) Shutdown market

b) Start market

14 Order Match System 
c) Suspend trading in $<x y z\rangle$

\section{Workflow}

Every "market command" is a workflow template enforcing a chain process through a synchronised sequence. These sequences are parallel with one thread for each instrument. The workflow bus walks the transaction through its phases very similar to the sub-processor micro-code instructions that make up a $\mathrm{CISC}^{15}$ processor (Sun Microsystems, 1993). Each template is composed of individual messages to market objects that the workflow subsystem enforces to either be executed in order, concurrently or a combination.

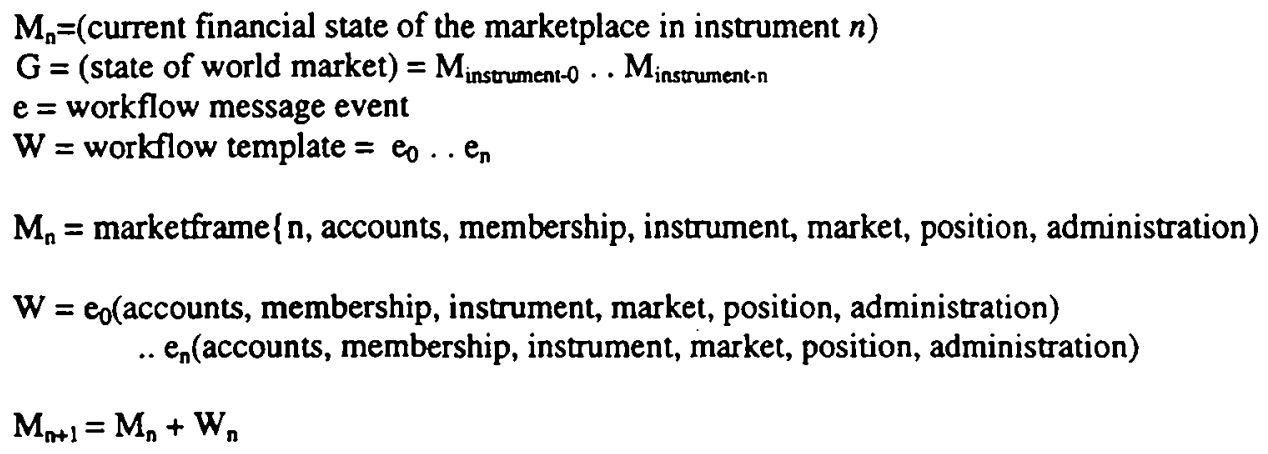

A workflow template is a type of service administrated in the administration object as the marketplace instruction template for a transaction. Example marketplace transaction:

ABC Company of South Africa entered the market (after having registered and established a connection prior) to BUY 100,000 shares of XYZ at 51 privately negotiated market", this indication of interest was routed by ABC preference, to two dealers, one, Cayman Islands Ltd., and the other, Frankfurt Mainz AG both seeing ABC's need, each with significant holdings in XYZ, they each reply "SELL 100,000 XYZ but the Cayman island dealer places a limit of 51.15 on his sell and the Frankfurt dealer's message matches with $5,100,000$. Post-match media services are contacted of the transfer, the ABC company has been debited 5,100,000 The Frankfurt dealer pulls up a statement showing his reduced balance of 100,000 shares of $\mathrm{XYZ}$ and a payment of $5,100,000$. The depository record in instrument now shows $\mathrm{ABC}$ Company as $2.5 \%$ owners in $\mathrm{XYZ}$.

\begin{tabular}{|c|c|c|c|c|}
\hline $\begin{array}{l}\text { membership } \\
\text { ABC Company }\end{array}$ & $\begin{array}{l}\text { position } \\
\text { media snapshot }\end{array}$ & $\begin{array}{l}\text { market } \\
\text { media subscribe }\end{array}$ & $\begin{array}{l}\text { account } \\
\text { media snapshot }\end{array}$ & 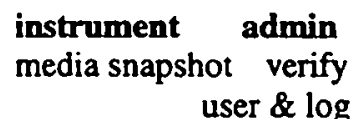 \\
\hline $\begin{array}{l}\text { ABC Company } \\
\text { ABC Company } \\
\text { ABC Company } \\
\text { Cayman Island }\end{array}$ & $\begin{array}{l}\text { Indication BUY } 100000 @ 51 \text { XYZ } \\
\text { Order BUY 100000@51 XYZ } \\
\text { xfer 5100K \& receive 100k XYZ } \\
\text { media snapshot }\end{array}$ & $\begin{array}{l}\text { implicit } \\
\text { implicit } \\
\text { order match } \\
\text { media subscribe }\end{array}$ & $\begin{array}{l}\text { implicit } \\
\text { implicit } \\
\text { implicit } \\
\text { media snapshot }\end{array}$ & $\begin{array}{ll}\text { implicit } & \log \\
\text { implicit } & \log \\
\text { implicit } & \log \\
\text { media snapshot } & \text { verify }\end{array}$ \\
\hline $\begin{array}{l}\text { Cayman Island } \\
\text { Frankfurt M. }\end{array}$ & $\begin{array}{l}\text { expire position } \\
\text { media snapshot }\end{array}$ & $\begin{array}{l}\text { Cancelled -outbid } \\
\text { media subscribe }\end{array}$ & $\begin{array}{l}\text { d implicit } \\
\text { media snapshot }\end{array}$ & $\begin{aligned} & \text { implicit } \log \\
& \text { media snapshot } \text { verify } \\
& \text { user \& log }\end{aligned}$ \\
\hline $\begin{array}{l}\text { Frankfurt M. } \\
\text { Frankfurt M. } \\
\text { XYZ Company }\end{array}$ & $\begin{array}{l}\text { Order SELL > } 100000 \text { shares of } X \\
\text { xfer } 100 \mathrm{XYZ} \& \text { receive } 5100 \mathrm{k} \\
-\end{array}$ & $\begin{array}{l}\text { YZimplicit } \\
\text { order match } \\
-\end{array}$ & $\begin{array}{l}\text { implicit } \\
\text { media snapshot }\end{array}$ & $\begin{array}{l}\text { implicit } \\
\text { implicit } \\
\text { ownership } \\
\text { change }\end{array}$ \\
\hline
\end{tabular}

\section{Marketplace Media Services}

The marketplace media support all requests for knowledge in the marketplace that do not involve changing the marketplace itself. These include historical price activity, position value-at-risk, year-to-date P\&L, accounts statements, quantitative views of derivative positions and the vast majority of the data on a current trading desk. Market media requests fall into two categories, snapshots and subscriptions. A snapshot is a single view of a market data image without updates for future changes even though it is on the screen. All paper reports in the

15 Complex Instruction Set 
financial industry today are snapshots. Subscriptions are continuously updating images of the market in a window on the user screen. As neither of these requests involves access to the central workflow bus, a media image can be generated from an integrated parallel reporting sub-system diagrammed in Figure 3 as "media services".

Snapshot media are similar to that supported current database access methods (Sybase, 1998). Subscription media are more complex due to the problem of simultaneous delivery of changes. If an order is matched, all subscriptions whose scope includes that portion of the market must be concurrently updated as discontinuous updates allow unfair advantage. This fan-out problem exists in today's markets, and is managed with high speed networking and intelligent design (NYSE, 1987).

\section{The bid-offer pool, a media design issue}

It is common practice for an exchange to quote the market as best bid \& offer price*size. A participant is inquiring into the media to find out what is the state of the market. Should I participate? How deep is the market? How fast can I execute this order without moving the market? How volatile is the instrument? A participant comes to the marketplace media with these questions. If the media describing the bid-offer pool is misleading, this benefits the "clever" participant over the innocent participant (Prindl, 1994). Depending on a media designer's ethical position, this microstructure asymmetry can deliberately or unconsciously influence the overall market's effectiveness (Shenk, 1997)(McLuhan, 1989). Marketplace media gives market participants the sensation of "being there" in the market and allows them to see and feel the state of the market. The effectiveness of the media interface is largely dependent on how well it uses currently-available technology to communicate the essence of the knowledge a participant needs to achieve their objectives. There is an argument for complete secrecy in a market to disguise large pension rebalancings and the dumping of blocks into the market. Entering the market with a large block can be difficult without secrecy as competitive bidders benefit from the disclosure of the strategy that one is forced to sell (Straffin, 1993). If real-time data costs drop regarding a market, does this reduced threshold increase participation? If the cost of participation is the ability to interpret and understand the market, does providing lucid media regarding the potential execution prices draw or diminish interests on willing participants to trade in the market? The issues presented are suggested merely to promote the ethical argument over whether such design should be completely opaque to the inquiring economist or studied and modelled.

\section{Profit \& loss}

When marking a position, there is some consideration as to the time-value of the marking if the market liquidity will not support that value within that timeframe. Consider the example of the firm with 200,000 corporate bonds seeking a marking price from the market to assess the value of the holding. The market for the bonds would fetch a price of 88 if sold over 1 week and a price of 93 if sold over 3 weeks due to market liquidity. P\&L Mark-to-Market ${ }^{16}$ pricing media should support mark-to-depth sensitivity as part of the incorporated P\&L media services to afford the users of an ideal market a realistic standard for marking assets. (Wurtz, 1998) The complexity of this area can be awe-inspiring. Risk and profitability media represent the cutting edge of financial engineering design and thus are unlikely to be easily incorporated into an open framework without significant forethought. The intent to include risk and volatility standards as services of the market opens up the ethical question of whether it is in the stakeholder's best interest to have these things be vague and un-standardized (Prindl, 1994)

Example media queries:

- Inquire history of account

- View Position

- View Position changes

- View P\&L marked to 1-month liquidation

- View Transactions

- View market in GBP/USD

- View Cash Flow P\&L's, Duration \& Convexity

- View Value-At-Risk for Account

- View Quantitative Valuation Modelling

- Duplicate Market

16 using the market assessment as the "price" and book value of the position 


\section{CAPITAL MARKETS EFFICIENCY AND EFFECTIVENESS MULTIPLIER}

Current macroeconomic theory is heavily entwined with the concept of free, relatively-frictionless capital markets. A simultaneous market yields a higher efficiency in the conversion of savings to investment (S-I) in macroeconomic modeling by reducing the transaction premium of the marketplace through reduced risk, reduced transaction cost and better information flow. Efficiency is improved by pure throughput of the capital market through the combination of more participants and more products introduced to each other on a vastly more efficient commerce medium than is in use today. Effectiveness would also be improved by better information flow and reduced short-term volatility leading to more intelligent expenditure and return on funds. This information flow also reduces the potential for hyperreal finance (McGoun, 1997) by reducing the opportunity for agency interests within the marketplace itself. Such considerations include the potential to perform macroeconomic adjustments through the introduction of simultaneous electronic markets.

\section{ASYMMETRIES \& CONSIDERATIONS}

Following are discussed several factors contributing to market asymmetries and some considerations in the tradeoff between frictionless objectives and current standard practices.

\section{Exchanges}

There was a celebration in the SIAC (NYSE's automation company) during one of my research interviews after the close of the largest equity market crash since 1987, October 13, 1989. It was explained on inquiry that the celebration was due to the record high transaction volume in the electronic systems during the market downturn and that that would increase the salary bonuses. The order-match system designers in this exchange have a motivation to design a market that pays their company well and that sustains their jobs building more systems that work within the exchange framework. They do not have a motivation to increase the wealth of the stakeholders in the market through efficient software microstructure design, nor do they have the liberty. They also have a strong corporate motivation to over-sell the complexity of market system design to guard against potential entrants to their business.

\section{Sovereign}

The question in marketplace architecture is should sovereign nations be treated like any other participant, selling and buying bonds for currency. Other matters regarding sovereign control are not electronic such as public disclosure, adherence to the rule of law, the privacy of physical records, and member policing.

\section{Accounting}

In terms of a financial market, all public accounting standards match a transaction log to the public record. They are completely important in describing assets through standards that are used in the public media of the market. In terms of our efficient market, accounting standards are a type of media view on assets. The accounting discipline is thus split in usage in this perfect market. Accounting as a matter of public record (Financial Accounting) and accounting as a discipline of measuring real value. The former of the account domain and the latter part of the position domain.

\section{Legal Issues}

In a simultaneous market, contract law is implicit in the market. Thus the technology of a simultaneous market is (under perfect conditions) law in itself legitimately transferring real ownership of goods and services in realtime. Parties can conflict and resolve using transactions, but transactions are never backed out. Though this seems trivial, it is an important assumption omitted in earlier work. The legal dominance of a sovereign entity over a marketplace is an implicit asymmetry toward the sovereign state. The market-domicile sovereign has the power to change the rule of law governing products owned and represented by parties foreign to the state. Since a real-time depository is considered in the ideal model, the ontology's described herein are legally binding contractual terms within the market. If legal challenges are allowed to tamper with past transactions in a depository marketplace, then all dealing parties will have to hedge the risk of market failure from legal action, undermining the legitimacy of a unchallenged depository market-record. 


\section{Backing out transactions}

In current operations, it is common for an account number to be mis-keyed by a clerk keying in a trader's ticket or for a ticket to be misplaced. Carry calculations and end-of-period dividend and tax calculations all involve having a proper transaction record at the time of calculation. When a transaction is applied "as-of" it requires the recomputation of the entire market subsequent to the transaction as a missed transaction changes FIFO P\&L matching, carry-cost calculations, depository transfers and such. Missed or incorrectly entered transactions 17 currently justify days of delay in proper bookkeeping If we keep with the premise of "perfect fit-to-function and failure-free" market software with user-impervious end screens, invalid keying can be dropped as a manualsystem side-effect. Since a very high percentage of incorrect or lost transactions are related to human involvement in the process in the first place, the ideal market guarantees internal integrity by having the endcustomer enter a transaction once, correctly, with no subsequent human involvement. The valid transaction is then part of a forward time rolling legal depository.

\section{Netting, Stock Borrow/Loan, Margins and Commissions}

Each of these areas of real-world markets usually involves a separate software environment and special treatment. Netting does not exist in a simultaneous market as each transaction clears individually. Stock Loan, margins and commissions are administrative sub-components of market transactions. As each component transaction is a market position in its own right, no separate consideration is given these as they can be effectively included within the architecture given by simply extending the workflow definition of a transaction.

\section{REFERENCES}

Arbnor, Ingeman; Bjerke, Bjorn (1997) Methodology for Creating Business Knowledge, Sage Publications, London UK

Barber, Rick (1997) TOREX ${ }^{\mathrm{TM}}$ trading station - Derivative Trading Functionality, Toronto Stock Exchange, Toronto, Ontario Canada

Bellcore 1981-1989 CCITT \#7 (Signal Switching protocol \#7) Protocol Message \& State design Bellcore Publications, New Jersey USA

Bernstein, Richard (1995) Portfolio Trading Guide, Part 1 - Adding Value Using Basket Trading Methods Merrill Lynch \& Co., Global Securities Research \& Economics Group, Quantitative \& Equity Derivatives Research Department, New York City, USA

Boyd, J.; Smith, B. (1997, April) Capital Market Imperfections, International Credit Markets, and Nonconvergence Journal of Economic Theory, vol. 73, no. 2, pp. 335-364

Cohen K., Maier et. al. (1986) The Microstructure of Securities Markets New Jersey: Prentice-Hall

CME - Chicago Mercantile Exchange; CBOT - Chicago Board of Trade (1997) Order Routing API Developer Guide CME \& CBOT Chicago USA

D’Sylva, Ray (1991) "Artificial Intelligence/GUI for dynamic service creation under SS7" NYNEX AI Technology Laboratories, White Plains, New York

Fabozzi, Frank; Modigliani, Franco (1996) Capital Markets Institutions and Instruments; Second Edition Prentice Hall, New Jersey USA

FIX - Financial Information Exchange (1998) www.fixprotocol.org

Freixas, Xavier; Rochet, Jean-Charles (1997) Microeconomics of Banking MIT Press, Cambridge, Mass. USA

Jang, Jeff (1998) ScotiaMcleod Equity Trading Platform (tables) Scotiabank internal, Toronto Ontario Canada

Kahin, Brian; Abbate, Janet (1995) Standards Policy for Information Infrastructure The MIT Press Cambridge Massachusetts USA pp. 378-615

Kam, Vernon (1990) Accounting Theory Second Edition, John Wiley \& Sons, New York USA pp. 8-30, 149152

Khalil, D. (1997) Universal Financial Transaction Protocol Discrete Arbitrage Nine LLC, New York USA

17 Human errors including switching off modems, power failures, programmer error, human clerk key entry error, incorrect receipt of clearing ticket, incorrect booking of error transaction by staff are all common errors happening every day in the industry each slowing down the pipeline of whatever marketplace transaction is in queue for system services. End-to-End media integration limits run-time errors to the market participants themselves by eliminating the source of the majority of errors, disparate, complex systems based on the accounting and international trade market of the cold war. 
KSE - Korea Stock Exchange (1996) Outline of Korean Stock Price Index Futures to be introduced in 1996 Korea Stock Exchange, Stock index futures market organising committee, Seoul, Korea

Marinelli, J (1991) BESS system: SWIFT security access architecture Westpac Bank, New York City USA Mill, John Stuart (1859) On Liberty Hackett Publishing Company, Inc. Indianapolis, Indiana USA

MIT (1992) X Window Protocol Definition The X Consortium MIT, Cambridge, Mass. USA

McGoun E.G. (1997, Feb.) Hyperreal Finance, Critical Perspectives on Accounting, Vol. 8, no. 1/2, pp. 97-122

McKendrick, Ewan (1990) Contract Law Macmillan Press Ltd., London, England

McLuhan, Marshall; Powers, Bruce R. (1989) The Global Village, Transformations in world life and media in the 21st century Oxford University Press, London UK

Nicolle, T. (1998) Business Plan for a typical Citadel Client, Demica Ltd. London, England

NYSE (1987) MDS - Market Data Systems, Test plan for weekend testing SIAC, 55 Water Street, New York USA

NYSE (1998) Common Message Switch (CMS) API Programmers Guide, SLAC Corp. 55 Water Street, New York, NY 10005 USA

O'Leary, K \& Facih, Odile, (1997) Equity Trading System Request for Proposal, Nikko Europe Plc., London UK V 1.0

OM Technology AB (1996) The OMnet Application Programmer's Interface Manual Stockholm, Sweden

Optimark Inc. (1998) www.optimark.com

Pardo, Robert (1992) Design, Testing, and Optimisation of Trading Systems John Wiley \& Sons Inc. New York USA

Prindl, Andreas R.; Prodham, Bimal (1994) Ethical Conflicts in Finance Blackwell, UK

Reddy, Michael T. (1995) Securities Operations; Second Edition New York Institute of Finance Press.

Rosenburg, Ellen (1987) Multibond System Design Securities Industry Automation Corporation, Metrotech Center, Brooklyn USA

Shenk, David (1997) Data Smog, Surviving the Information Glut Harpercollins, New York USA

Smith, Adam (1776) The Wealth of Nations Prometheus Books, New York USA pp. 377

Straffin, Phillip D. (1993) Game Theory \& Strategy The Mathematical Association of America, Washington DC, USA

Sun Microsystems (1993) SunShip, Queue Management and Workflow Design Sun Microsystems Computer Corporation, Milpitas, California USA

Susskind, Richard (1996) The Future of Law; Facing the Challenges of Information Technology, Clarendon Press, Oxford UK

Sprint (1988) TTSI - DSP-MS accountcode interface specification US Sprint development laboratories, Burlingame, California USA

Sybase Inc. (1994) Open ClientTM and Open ServerTM Common Libraries Reference Manual Emeryville, California USA

Tandem Computers (1989) TNS-II interprocessor bus and the non-stop architecture, Tandem Computers Inc., Cuptertino California USA

Wolfe, Nancy (1988) E911-MS, Error Processing for service order parsing failures Pacific Bell Telephone Corp., San Ramon, California USA

Wurtz, Charles (1998) "Marking for depth", Veristics Inc., New York USAhttp://www.optimark.com/

Xticket Systems Inc. (1995) Documentation of the Physical Data Model, Position Database (Xticket Internal) Manhattan, New York USA

\section{ACKNOWLEDGEMENTS}

This paper is dedicated to the memory of Dr. Frederick Lenz.

So much depends on the red wheelbarrow in the rain next to the white chickens.

This research has supported by the following:

- Sybase Inc., London \& Toronto

- The Royal Bank of Canada, Toronto

- Scotia Capital Markets, Toronto

- Reuters America, New York
- Petra Kloppenburg, Edinburgh

- Dr. Charles Wurtz, New York 


\section{Composition of media}

\section{APPENDIX A}

Each market participant engages in their connection(s) with the electronic marketplace via network media services. From the point of view of the digital market, all participants are merely electronic messages requesting services that it subsequently provides. Although the actual substance of the media can vary widely depending on the participant and their interactions with the marketplace, the types of media modalities are few. A physical network of adequate bandwidth to support multiple video, audio \& digital data multicast and point-to-point communications is required.

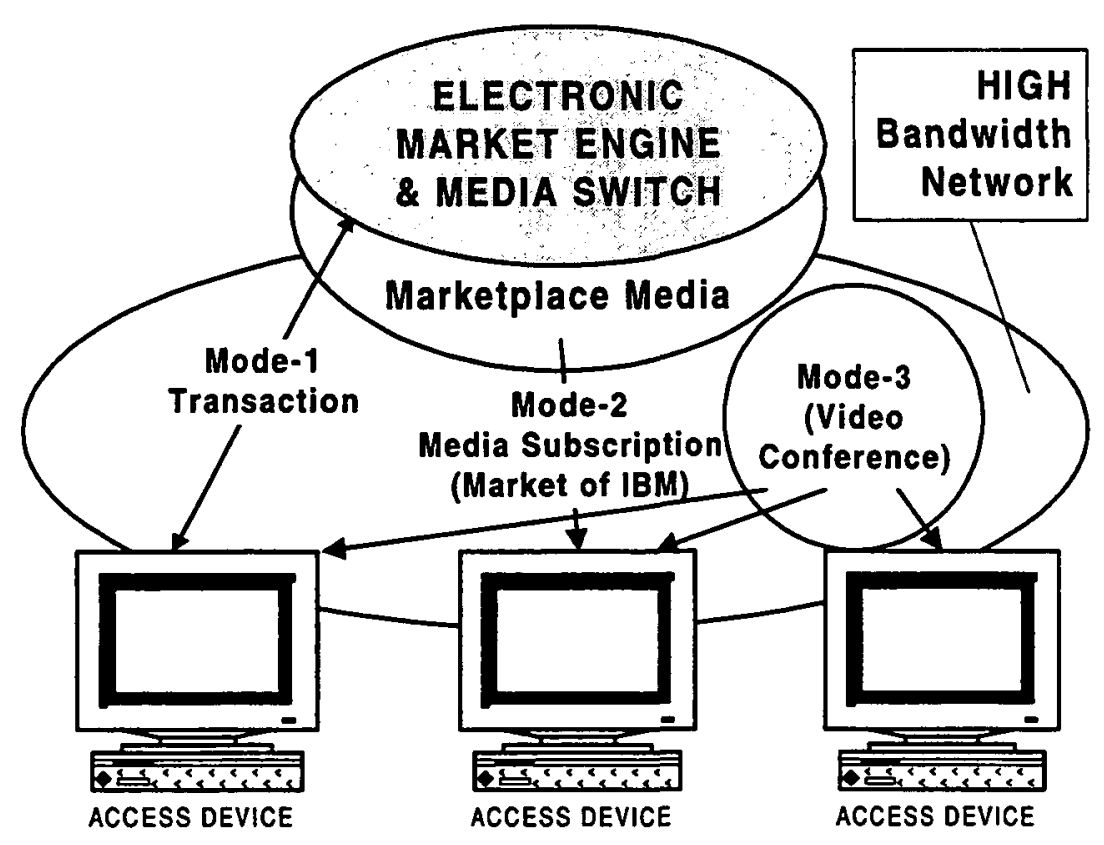

Figure 5. Shown are the three modes of media services within this electronic market. The diagram parts can be related to the CCITT\#7 Service Control Point, Signal Transfer Point and switch with a high bandwidth local loop.

\section{Access devices}

The device that a user employs to engage a market (via its media services) incorporates bi-directional audio (telephone, orders), video (television, video-conference), text (news and interactive services) An X-Window (MIT, 1994) paradigm of end-user windows each driven by a media stream is adequate. The technology of switching media between windows is well researched in the operation of the current public Internet.

\section{Switching services}

The marketplace must provide a mechanism for the network to dynamically change itself as membership changes and to engage any media modalities with any access point in the network. This switching service is supported and made available via a command center that is instructed by software to engage various media between various points from instructions in a dynamic database/expert system. This paradigm is better supported by an extension of the (Bellcore, 1988) Signalling System \#7 protocol ${ }^{18}$ (Sprint, 1988) than the current web technology due to the separation of the signalling network from the "voice" or data network within the telephone network and protocol-integrated load balancing. Also because signalling information shares bandwidth with data on the Internet and because no reliable point-to-point services can be ensured, the medium is a weak standard for this virtual marketplace.

18 The functionality described in this paper is programmable using current commercial technology between a hybrid multimedia telephony switch, an upgraded signal transfer point, a supercomputer service control point and a high-bandwidth global network. The entire switching engine would reside in the service control point operating a global market. The service control point is itself a virtual network intelligence engine simultaneously replicated to other sites via an "inter-processor bus" (Tandem, 1996). 
The telephony paradigm is also selected to overcome a difficult hurdle in current financial operations and internetworking, weak transaction reference data. When using a current open connectivity standard for financial interconnection such as (FIX, 1998) ${ }^{19}$, the homogeneity of the reference data cannot be guaranteed and is accepted as weak. When interconnecting with 10 dealers, the problem is manageable. When scaled to 10,000 dealers, the problem is unmanageable and the standard breaks down. In telephony, all connections use the internal network databases, and system services are both hardware and application fault-tolerant. It is then possible for the telephone company to guarantee the quality of their services due to this closed architecture and the same must be true in an ideal market.

\section{Modality}

Any communications link in the market is via one of three types of modalities. They each represent three fundamentally different communication services required in a virtual market. This paper really only is concerned with the first mode as switching and transmission technologies for the latter two are in use today. However, it is important to recognise the latter two as they make up the vast majority of marketplace services visible today with the first mode an opaque process within the dealing community.

1. Multi or single part discrete transaction. This channel requires guaranteed message delivery in a point-topoint fashion. The transaction is always initiated by the end user access device. It is composed of discrete packets of information that dialog between the source and the destination. (e.g. "what happened in account 123 last month")

2. Subscription. The channel requires information to be subscribed to until a request is made to discontinue the subscription. The data are delivered as a packetized stream of whatever media composition is requested. (show the active IBM open order book, watch CNN television) (1998) Reuters SSL documentation

3. Multi or single message full-duplex streaming connection. This channel requires point-to-point connection services for omni-directional data communications until terminated. (e.g. telephone call or 5-way video conference)

As is evident in figure 4, the marketplace entities must be able to complete every desired transaction with the marketplace via these marketplace media devices (Workstations). The first type of media is in all cases a request from the signalling network for intelligent services, being it address resolution on an Email to placing an order into the market for XYZ. It is the focus of the technology in the paper as the latter two media systems are already standardised.

19 each user must maintain the logical address database of all interconnected parties 